\title{
Integrative Network Analysis Reveals a MicroRNA-Based Signature for Prognosis Prediction of Epithelial Ovarian Cancer
}

\author{
Li Li, ${ }^{1}$ Haiyan Gu, ${ }^{1}$ Lingying Chen, ${ }^{1}$ Ping Zhu, ${ }^{2}$ Li Zhao, ${ }^{3}$ Yuzhuo Wang, ${ }^{4}$ Xiang Zhao, ${ }^{5}$ \\ Xingguo Zhang $\left(\mathbb{\infty},{ }^{6}\right.$ Yonghu Zhang $\left(\mathbb{0},{ }^{7}\right.$ and Peng Shu $\oplus^{6}$ \\ ${ }^{1}$ Department of Gynecology, Beilun People's Hospital, Ningbo 315800, China \\ ${ }^{2}$ Department of Gynecology, Cangzhou People’s Hospital, Cangzhou 061000, China \\ ${ }^{3}$ Department of Urology, Hefei BOE Hospital, Hefei 230041, China \\ ${ }^{4}$ Prenatal Diagnostic Laboratory, Cangzhou People’s Hospital, Cangzhou 061000, China \\ ${ }^{5}$ Department of Hepatobiliary Surgery, Nanjing Jiangbei People's Hospital Affiliated to Nantong University, Nanjing 210048, China \\ ${ }^{6}$ Department of Clinical laboratory, Beilun People's Hospital, Ningbo 315800, China \\ ${ }^{7}$ Department of Infectious Diseases, Beilun People's Hospital, Ningbo 315800, China
}

Correspondence should be addressed to Xingguo Zhang; xgzhang666@163.com, Yonghu Zhang; 46587018@qq.com, and Peng Shu; m17757498873@163.com

Received 9 January 2019; Accepted 18 April 2019; Published 4 June 2019

Academic Editor: Stephen H. Safe

Copyright (C) $2019 \mathrm{Li} \mathrm{Li}$ et al. This is an open access article distributed under the Creative Commons Attribution License, which permits unrestricted use, distribution, and reproduction in any medium, provided the original work is properly cited.

\begin{abstract}
Background. Epithelial ovarian cancer (EOC) is a heterogeneous disease, which has been recently classified into four molecular subtypes, of which the mesenchymal subtype exhibited the worst prognosis. We aimed to identify a microRNA- (miRNA-) based signature by incorporating the molecular modalities involved in the mesenchymal subtype for risk stratification, which would allow the identification of patients who might benefit from more rigorous treatments. Method. We characterized the regulatory mechanisms underlying the mesenchymal subtype using network analyses integrating gene and miRNA expression profiles from The Cancer Genome Atlas (TCGA) cohort to identify a miRNA signature for prognosis prediction. Results. We identified four miRNAs as the master regulators of the mesenchymal subtype and developed a risk score model. The 4-miRNA signature significantly predicted overall survival (OS) and progression-free survival (PFS) in discovery $(p=0.004$ and $p=0.04)$ and two independent public datasets (GSE73582: OS, HR: 2.26 (1.26-4.05), $p=0.005$, PFS, HR: 2.03 (1.34-3.09), $p<0.001$; GSE25204: OS, HR: 3.07 (1.73-5.46), $p<0.001$, PFS, HR: 2.59 (1.72-3.88), $p<0.001)$. Moreover, in multivariate analyses, the miRNA signature maintained as an independent prognostic predictor and achieved superior efficiency compared to the currently used clinical factors. Conclusions. In conclusion, our network analysis identified a 4-miRNA signature which has prognostic value superior to currently reported clinical covariates. This signature warrants further testing and validation for use in clinical practice.
\end{abstract}

\section{Introduction}

Epithelial ovarian cancer (EOC) is the most lethal gynecological malignancy, characterized by molecular and pathological heterogeneity. The main pathological type is the high-grade serous ovarian cancer (HGSOC) [1]. Despite new screening and treatment strategies, the prognosis for EOC remains poor. The main reason for the poor prognosis is late-stage presentation during diagnosis, widespread dissemination, and high recurrence rate [2]. Standard treatment for EOC is aggressive resection of the primary tumor followed by adjuvant chemotherapy. Owing to the diffuse nature of EOC, the relapse rate is high even in those who initially had a complete pathological response, and most of the patients developed chemoresistance [3]. Overall survival (OS) has improved moderately over the past 30 years, with a 5 -year survival rate of approximately $30 \%$ [4].

Several studies have attempted to develop molecular signatures based on gene expression to predict EOC prognosis. However, only few molecular prognostic signatures have been developed [5-7], of which not many have been externally verified, and none of them could be directly applied in 
TABLE 1: Patient characteristics.

\begin{tabular}{lccc}
\hline & $\begin{array}{c}\text { Training cohort } \\
\text { (TCGA, } n=462)\end{array}$ & $\begin{array}{c}\text { Validation cohort } \\
(\text { GSE73582, } n=133)\end{array}$ & $\begin{array}{c}\text { Validation cohort } \\
(\mathrm{GSE25204,} n=130)\end{array}$ \\
\hline $\begin{array}{l}\text { Age (years) } \\
\text { Stage }\end{array}$ & $60(30-87)$ & $56(27-82)$ & $54(25-85)$ \\
I & & $16(12 \%)$ & $9(7 \%)$ \\
II & $21(5 \%)$ & $105(79 \%)$ & $23(18 \%)$ \\
III & $361(78 \%)$ & $3(2 \%)$ & $1(1 \%)$ \\
IV & $76(16 \%)$ & & $25(19 \%)$ \\
Unknown & $4(1 \%)$ & $5(4 \%)$ & $95(73 \%)$ \\
Grade & & $29(22 \%)$ & $2(2 \%)$ \\
1 & $55(12 \%)$ & $82(62 \%)$ & $17(13 \%)$ \\
2 & $394(85 \%)$ & & $21(16 \%)$ \\
3 & $14(3 \%)$ & $55(41 \%)$ & $109(84 \%)$ \\
Unknown & & $77(58 \%)$ & $1(1 \%)$ \\
Debulking & $302(65 \%)$ & & \\
optimal & $112(24 \%)$ & & \\
suboptimal & $48(10 \%)$ & & \\
Unknown & & & \\
\hline
\end{tabular}

clinical practice. One of the reasons for the low prognostic efficacy is the heterogeneity of EOC during initial diagnosis. Recently, Tothill et al. [8] identified 4 subtypes of ovarian cancer with distinct molecular and clinical characteristics by unsupervised classification of the gene expression patterns and revealed that the mesenchymal subtype had the worst OS. Subsequently, the mesenchymal subtype was recapitulated in several other studies $[9,10]$. Therefore, exploring the underlying determinants of the poor prognosis mesenchymal subtype could be potentially used for risk stratification and for developing more precise, targeted treatment strategies for EOC patients.

To find a better risk prediction method for EOC patients, we decided to focus on miRNAs-a class of short sequences of noncoding RNA [11], since they act as the master regulators of gene expression [12]. In this study, we applied network analysis to reveal the regulatory mechanisms underlying the mesenchymal subtype, integrating gene, and miRNA expression profiles. The master regulator analysis showed that the mesenchymal subtype was regulated by four miRNAs.

Although studies on miRNA signatures in EOC have been reported $[13,14]$, no research has been done by integrating the characteristics of molecular subtypes for risk stratification. We aimed to identify a miRNA-based signature by incorporating a variety of molecular modalities involved in the mesenchymal subtype for risk stratification that would allow the identification of EOC patients who might benefit from more rigorous treatments.

\section{Materials and Methods}

2.1. Patient Series. In this retrospective study, we performed a comprehensive analysis of 725 patients using three independent miRNA cohorts from women with EOC (Table 1). These cohorts included patients from the
TCGA dataset (training cohort, $\mathrm{N}=462$ ) [9], the GSE73582 cohort (validation set, $\mathrm{N}=133$ ) [15], and the GSE25204 cohort (validation set, $\mathrm{N}=130$ ) [16]. mRNA profiles were derived from the TCGA dataset consisting of 462 EOC patients [9]. The expression profiles of miRNAs and mRNAs together with the corresponding clinicopathological parameters were downloaded from Gene Expression Omnibus (GEO http://www.ncbi.nlm.nih.gov/geo/) and TCGA data portal (https://tcga-data.nci.nih.gov/tcga/). The study was performed in accordance with the Declaration of Helsinki and approved by the institutional review board of Beilun People's Hospital, Ningbo, China.

2.2. Expression Data Preprocessing. Microarray data of the two validation cohorts, normalized using the robust multiarray analysis (RMA), were downloaded from GEO using R package "GEOquery" (version 1.0.7) [17]. We further removed the nonbiological batch effects of the GSE25204 cohort using ComBat function in R package "sva". The Cancer Genome Atlas (TCGA) dataset, including 462 matched miRNA and mRNA expression profiles, was downloaded from the TCGA data portal by "TCGAbiolinks" R package [18] and normalized using voom function in limma R package [19]. For each dataset, the expression profiles were transformed from probe sets or entrez sets to gene symbols.

2.3. Regulatory Network Inference. We have applied regulatory network inference to study the regulatory mechanisms of the mesenchymal subtype by integrative analysis of miRNAs and mRNA expression profiles. Together, we analyzed 462 patient samples with both miRNA and mRNA expression profiling data in the TCGA cohort. Thirty-six miRNAs (|log2 fold change| $>0.5$, Benjamini-Hochberg adjusted $p<0.05$ ) and 1659 genes ( $\mid \log 2$ fold change| $>0.25$, BenjaminiHochberg adjusted $p<0.05)$ were differentially expressed 
in the mesenchymal subtype compared with the other three subtypes. The miRNA and mRNA expression profiles were normalized independently and subsequently integrated for network inference in the R "RTN" package [20]. We performed master regulator analysis (MRA) [21] to test epithelial-mesenchymal transition (EMT) genes significantly overrepresented in each miRNA's regulon. Four microRNAs of top significance (Benjamini-Hochberg-adjusted $p<0.05$ ) were selected as the master regulators of the mesenchymal subtype.

2.4. miRNA Signature Construction and Risk Score Calculation. Four miRNAs were differentially expressed in the poor prognosis subtype and were identified as the powerful determinants of the mesenchymal-specific gene expression, including EMT-related genes. Based on these four miRNAs, a cox-model was established as follows: risk score $=(0.0685 \times \mathrm{miR}-449 \mathrm{a})+(0.1258 \times \mathrm{miR}-409-3 \mathrm{p})+(-$ $0.0081 \times$ miR-200a $)+(-0.1176 \times$ miR-508-3p).

2.5. Statistical Analysis. Wilcoxon signed-rank test was used to identify differentially expressed miRNAs between different groups. Patients were dichotomized into high and low-risk groups by the median value of risk scores. The Kaplan-Meier curves were plotted to compare the survival analysis by using a log-rank test. Additionally, univariate and multivariate cox proportional hazard regression models were used to calculate hazard ratios to evaluate the prognostic significance of clinicopathological variables and the 4-microRNA signature. Statistical significance was denoted by $* p<0.05, * * p<0.01$, and $* * * p<0.001$, and a $\mathrm{p}$ value of less than 0.05 was considered significant. All statistical analyses were performed using R (versions 3.4.0, https://cran.r-project.org/).

\section{Results}

3.1. Integrative Analysis Identifies Master miRNA Regulatory Network for the Mesenchymal Subtype. EOC is a heterogeneous disease, which has been classified into four molecular subtypes with distinct molecular and clinical characteristics, of which the mesenchymal subtype was found to exhibit the worst prognosis [8-10]. To investigate the regulatory mechanisms underlying the mesenchymal subtype of EOC, we generated regulatory networks by integrative analysis [20] of miRNA and mRNA expression profiles in the TCGA cohort (Figure S1). The miRNA networks consist of miRNAs that are differentially expressed in the mesenchymal group compared with the other three subtypes (Figure 1(a)) and were found to regulate the expression of most of the mesenchymalspecific genes. Master regulator analysis (MRA) revealed four microRNAs (miR-449a, miR-409-3p, miR-200a, and miR$508-3 p)$ as the dominant regulators in the mesenchymal subtype (Table S1), whose expression levels differ significantly between the mesenchymal and other three subtypes (Figure 2(b)). As reported previously, overexpression of the miR-200a [22], miR-449a [23], and miR-508-3p [24] is associated with the inhibition of the EMT program, whereas the high expression of the miR-409-3p [25] promotes tumor growth and the EMT program. EMT signature genes are significantly correlated with the four miRNAs, revealing that the mesenchymal property is indeed regulated by these four miRNAs (Figure 1(c) and Figures S2-3). Therefore, these four miRNAs are major regulators of the mesenchymal phenotype and can be potentially used for risk assessment of EOC tumors.

3.2. Development of a 4-miRNA Signature in EOC Patients. Using the TCGA cohort, we constructed a cox-model based on the expression of these four miRNAs: Risk Score $=(0.0685$ $\times$ expression value of miR-449a $)+(0.1258 \times$ expression value of miR-409-3p) + (-0.0081 $\times$ expression value of miR$200 a)+(-0.1176 \times$ expression value of miR-508-3p). Of the four miRNAs, 3 were associated with improved prognosis (miR-200a, miR-449a and miR-508-3p) and one with worse prognosis (miR-409-3p). Using the 4-miRNA signature, risk scores were calculated in the TCGA cohort. Patients were significantly divided into high- and low-risk groups (median risk score as cut-off value; Table S2) in terms of OS (HR $1.44,95 \%$ CI 1.12-1.85; $p=0.0037$; Figure 2(a)) and PFS (HR 1.31 , 95\% CI 1.01-1.70; $p=0.04$; Figure 3(a)). High-risk group patients showed a shorter median survival than did the lowrisk group (29 months versus 31 months).

3.3. Validation of the 4-miRNA Signature. To confirm the prognostic power of the 4-miRNA signature, risk scores were calculated, and patients were stratified into two risk groups (Table S2) in two publicly available validation cohorts. In GSE73582 and GSE25204, patients were significantly divided into high- and low-risk groups in terms of OS (GSE73582: HR 2.26, 95\% CI 1.26-4.05, $p=0 \cdot 005$, Figure 2(b); GSE25204: HR 3.07, 95\% CI 1.73-5.46, $p<0.001$, Figure 2(c)) and PFS (GSE73582: HR 2.03, 95\% CI 1.34-3.09, $p<0.001$, Figure 3(b); GSE25204: HR 2.59, 95\% CI 1.72-3.88, $p<0.001$, Figure 3(c)). In the univariate analysis, advanced stages and debulking status were significantly associated with prognosis; however, the 4-miRNA signature outperformed these clinical factors (Table 2). In GSE73582, the HR was 2.04 (95\% CI 1.34-3.10, $p=0 \cdot 0008$ ) and, in GSE25204, HR was 2.59 (95\% CI 1.72-3.88, $p<0.0001)$. Moreover, multivariate analysis demonstrated the 4-miRNA signature as the strongest predictor in two validation cohorts after adjusting for other clinical factors (Table 2)

3.4. An Association between the 4-miRNA Signature and Chemotherapy Response. To examine the association of the 4-miRNA signature with response to first-line platinumbased therapy [26], we analyzed the chemotherapy response within different risk groups in the GSE25204 cohort and identified an association between the 4-miRNA signature and chemotherapy response $(p<0.001$, Fisher's exact test; Figure 4). Low-risk group patients exhibited a high likelihood of platinum sensitivity and those patients in high-risk group tended to have a high likelihood of platinum resistance or partial platinum sensitivity. In the high-risk group and lowrisk group, $30.7 \%$ and $75.3 \%$ patients, respectively, achieved platinum sensitivity. 


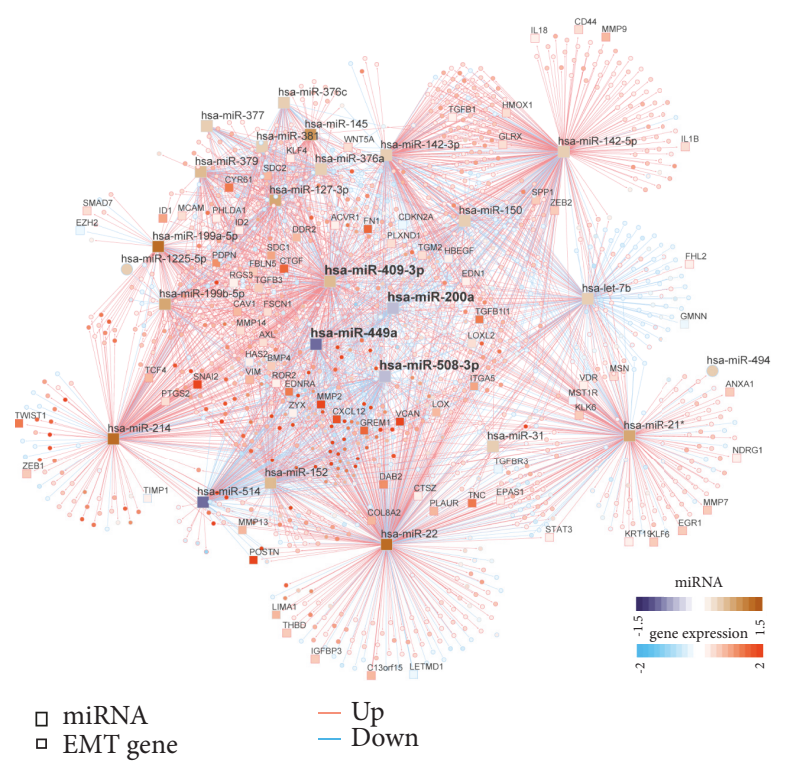

(a)
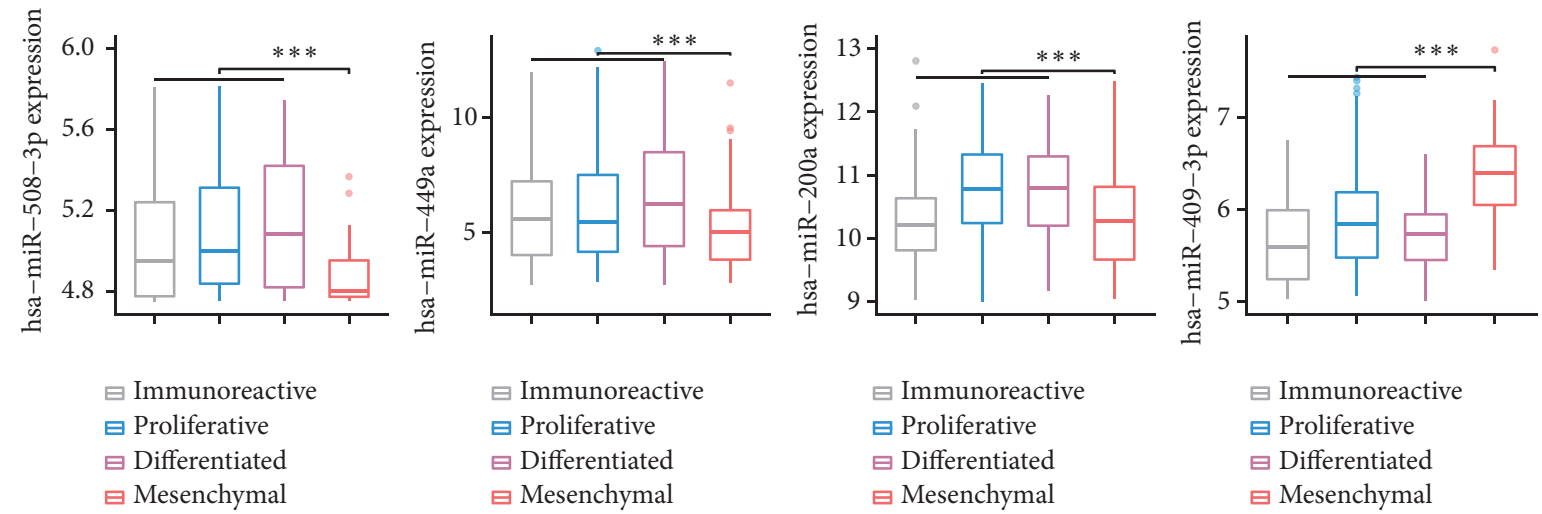

$$
\begin{aligned}
& \boxminus \text { Immunoreactive } \\
& \boxminus \text { Proliferative } \\
& \boxminus \text { Differentiated } \\
& \boxminus \text { Mesenchymal }
\end{aligned}
$$
$\boxminus$ Immunoreactive $\boxminus$ Proliferative
$\boxminus$ Differentiated $\boxminus$ Mesenchymal

$$
\begin{aligned}
& \boxminus \text { Immunoreactive } \\
& \boxminus \text { Proliferative } \\
& \boxminus \text { Differentiated } \\
& \boxminus \text { Mesenchymal }
\end{aligned}
$$

(b)
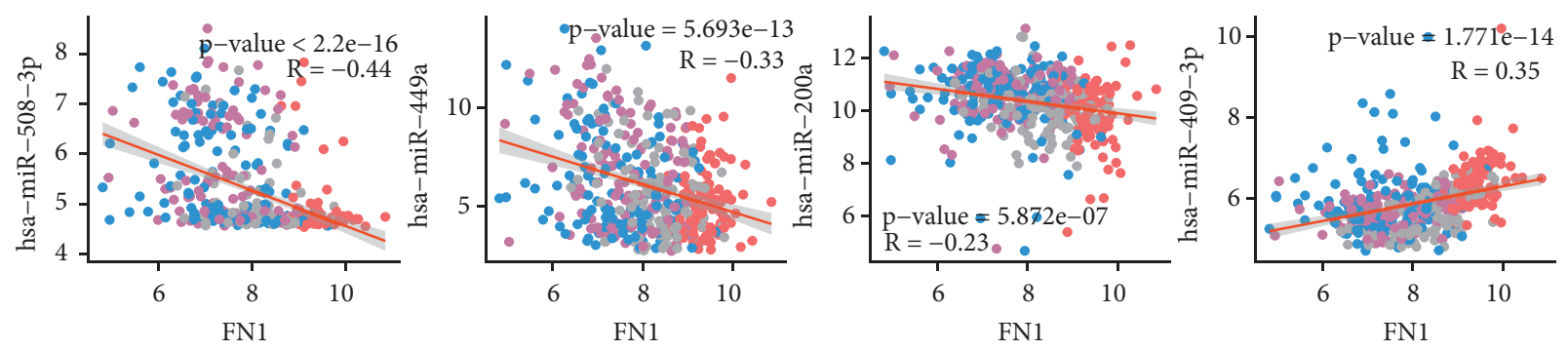

- Immunoreactive

- Immunoreactive

- Proliferative

- Proliferative

- Immunoreactive

- Immunoreactive

- Differentiated

- Differentiated

- Proliferative

- Proliferative

- Mesenchymal

- Mesenchymal

- Differentiated

- Differentiated

- Mesenchymal

(c)

FIGURE 1: Network inference analysis reveals four major regulatory networks of the mesenchymal subtype. (a) The mRNA-miRNA network shows the relationships between four key miRNAs and the EMT signature genes. (b) The four-miRNA signature was significantly lower in the mesenchymal subtype in the TCGA dataset than the other three subtypes. (c) Significant correlation between FN1 expression and the four-miRNA expression in the TCGA dataset. 
TABLE 2: Univariate and multivariate analysis.

\begin{tabular}{lcccccccc}
\hline & \multicolumn{3}{c}{ Validation Cohort (GSE73582) } & & \multicolumn{3}{c}{ Validation Cohort (GSE25204) } \\
& Univariate & Univariate & Multivariate & & Multivariate \\
& HR (95\% CI) & $P$ & HR (95\% CI) & $P$ & HR (95\% CI) & $P$ & HR (95\% CI) & $P$ \\
\hline Age (<65 vs. >=65) & $1.51(0.94-2.43)$ & 0.08 & $1.33(0.82-2.16)$ & 0.24 & $0.88(0.54-1.42)$ & 0.6 & $0.86(0.53-1.41)$ & 0.55 \\
Grade (3 vs. 1\&2) & $1.42(0.92-2.18)$ & 0.11 & $1.21(0.78-1.87)$ & 0.39 & $0.89(0.58-1.38)$ & 0.62 & $0.90(0.58-1.40)$ & 0.65 \\
Stage (III\&IV vs. I\&II) & $2.57(1.44-4.61)$ & 0.001 & $2.57(1.39-4.73)$ & 0.002 & & & & \\
Debulking (optimal vs. suboptimal) & $2.17(1.37-3.44)$ & 0.001 & $1.81(1.12-2.91)$ & 0.01 & $2.19(1.45-3.32)$ & 0.0001 & $1.80(1.17-2.77)$ & 0.007 \\
miRNA predictor (high vs low risk) & $2.04(1.34-3.10)$ & 0.0008 & $1.82(1.17-2.81)$ & 0.007 & $2.59(1.72-3.88)$ & $4.46 \mathrm{E}-06$ & $2.23(1.46-3.41)$ & 0.0002 \\
\hline
\end{tabular}

TCGA

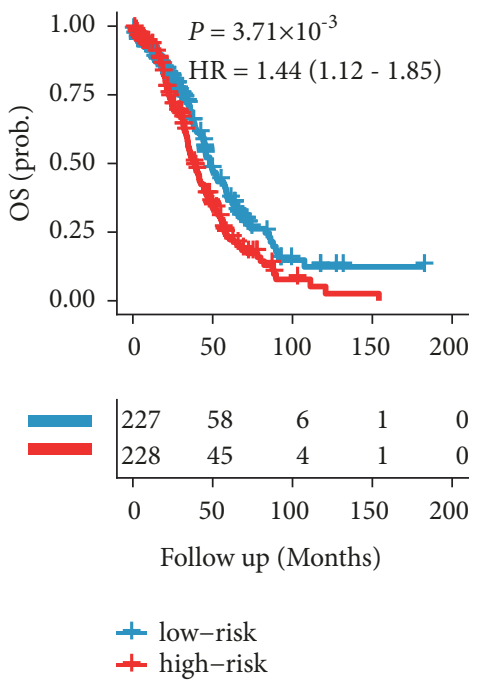

(a)
GSE73582

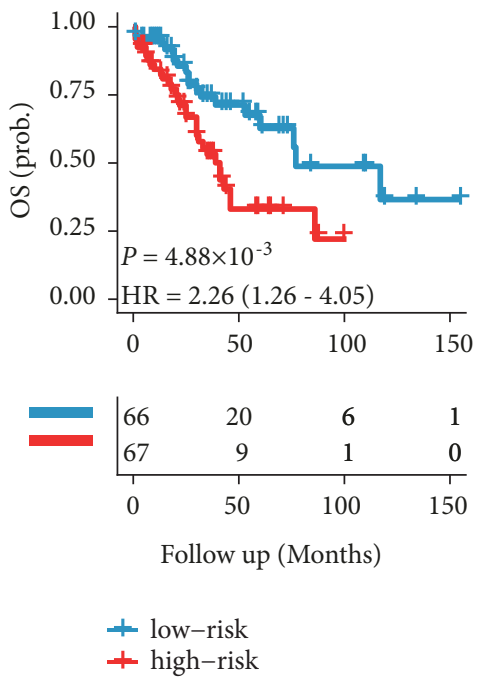

(b)
GSE25204

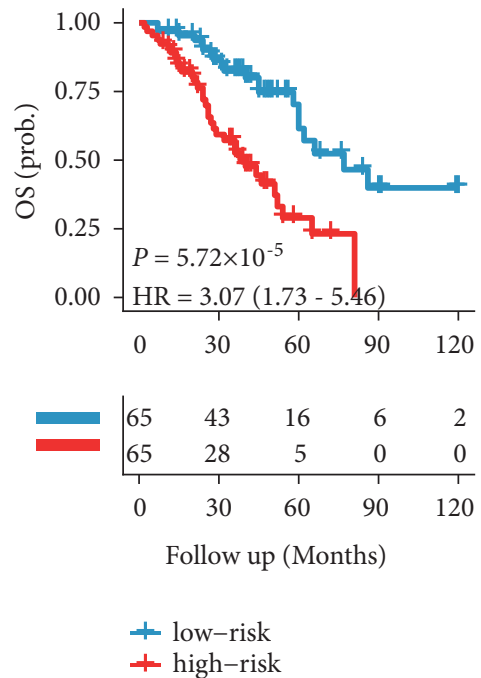

(c)

FIGURE 2: OS stratified by risk according to the 4-miRNA signature. The Kaplan-Meier plots show OS in patients stratified by the 4-miRNAs signature in the TCGA training cohort (a), GSE73582 validation set (b), and GSE25204 validation set (c). p values are based on log-rank tests.

TCGA

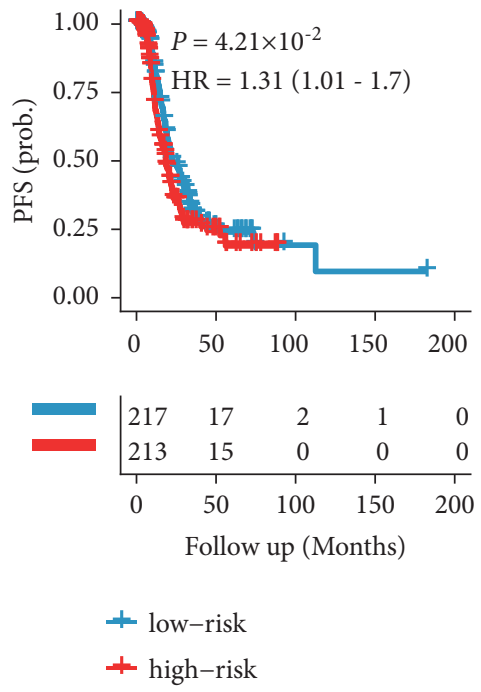

(a)
GSE73582

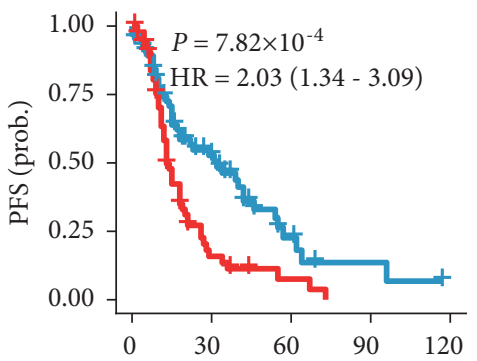

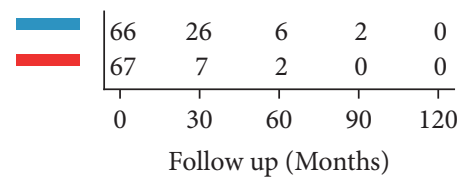

+ low-risk

+ high-risk

(b)
GSE25204
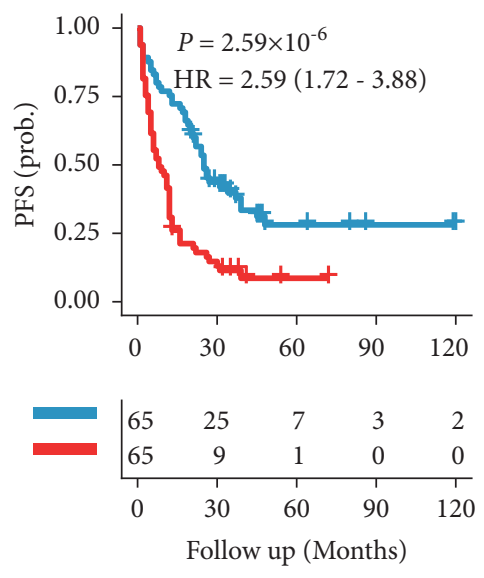

+ low-risk

+ high-risk

(c)

FIGURE 3: PFS stratified by risk according to the 4-miRNA signature. The Kaplan-Meier plots show PFS in patients stratified by the 4-miRNAs signature in the TCGA training cohort (a), GSE73582 validation set (b), and GSE25204 validation set (c). p values are based on log-rank tests. 


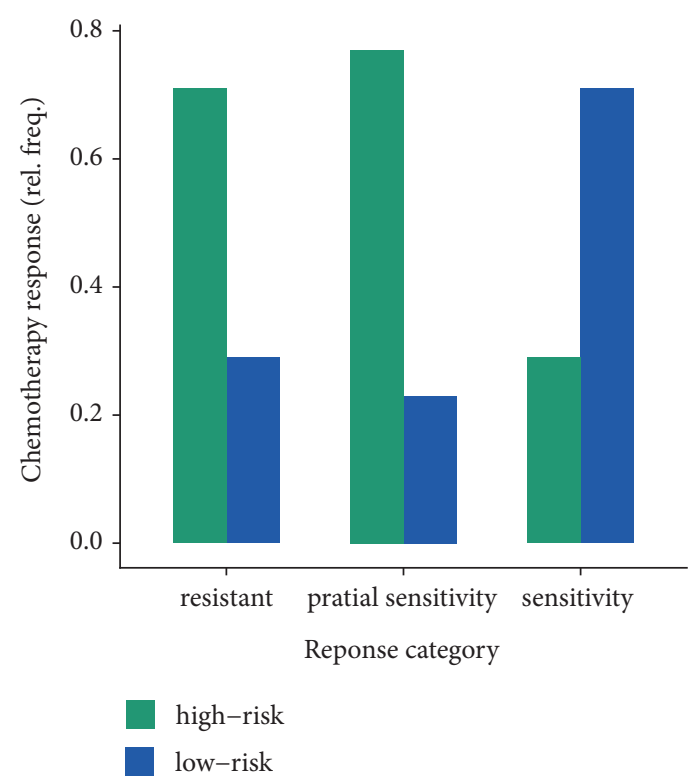

FIGURE 4: The association between the 4-miRNA signature and chemotherapy response.

\section{Discussion}

EOC is the leading cause of gynecological cancer deaths. Currently, clinical features such as tumor grade, histopathological classification, debulking status, and CA-125 levels are the most common criteria to evaluate the risk of HGSOC patients [27]. Although a lot of multigene prognostic signatures [9, 28-30] have been developed, the accuracy of their prognostic prediction remains uncertain. A method to identify EOC patients with a worse prognosis is urgently needed to improve the design of customized therapies.

Unsupervised classification of EOC transcriptome profiling revealed four molecular subtypes with distinct molecular and clinical characteristics [8-10]. The subtype-specific molecular portraits, especially the worst prognosis subtypespecific prognostic signature, could be potentially used for risk stratification $[24,31]$. We aimed to build a widely useful signature that integrates the molecular differences seen in the poor prognosis subtype of EOC. miRNAs are short noncoding RNAs that regulate gene expression and have been demonstrated as prognostic biomarkers in EOC [32]. The development of the miRNA-based signature is based on network analysis to identify a variety of molecular modalities involved in the mesenchymal phenotype. Our 4-miRNA signature revealed the regulatory mechanisms of the mesenchymal subtype and was able to identify groups of patients with significantly poor OS and PFS. The high-risk group patients had a worse prognosis and exhibited poor response to chemotherapy, suggesting that more aggressive treatments would benefit them. The 4-miRNA signature maintained its independent prognostic power in multivariate analysis after adjusting for tumor stage and debulking status, which are established clinical factors for prognostic estimation of EOC patients.
All the four miRNAs have already been reported as having fine-tuning roles in EMT processes. Of the four miRNAs, three contribute to a favorable prognosis and one contributes to worse prognosis. Zhao and colleagues identified that miR508-3p was involved in the regulatory network related to the EMT program in the mesenchymal subtype of EOC. Higher expression of miR-508-3p was associated with significantly better prognosis [24]. Chen et al. demonstrated that overexpression of miR-449a inhibited tumor metastasis and was associated with better disease-free survival of patients with hepatocellular carcinoma (HCC) [23], suggesting its oncosuppressive role. $\mathrm{Lu}$ et al. have reported that loss of miR-200a expression is associated with the EMT phenotype and promoted cell migration and invasion in pancreatic cancer [33]. However, elevated expression of miR-409-3p was observed in metastatic prostate cancer and was correlated with poor PFS [25]. The main prognostic effect of the 4miRNA signature was associated with the regulation of the EMT program, which initiates tumor spread and progression of EOC.

Three independent cohorts with mature follow-up information were used to construct a prognostic predictor for EOC. Since we focused on the analysis of miRNAs shared by all the platforms, we may have missed other meaningful miRNAs. Before the 4-miRNA signature can be developed for routine clinical use, its limitations should be considered. First, the prognostic signature is based on expression profiles produced by microarray platforms, which are difficult to popularize for routine clinical use due to their high price, long conversion cycle, and requirement of bioinformatics expertise. Second, more datasets with full clinical annotations need to be included in the analysis for broader validation.

\section{Conclusions}

In conclusion, our network analysis identified a 4-miRNA signature which has prognostic value superior to currently reported clinical covariates. Our study represents the first attempt to integrate tumor heterogeneity and develop a risk model which could be validated in silico.

\section{Data Availability}

The data used to support the findings of this study are available from the corresponding author upon request.

\section{Conflicts of Interest}

All authors declare that they have no conflicts of interest.

\section{Authors' Contributions}

Li Li, Haiyan Gu, and Lingying Chen contributed equally to this work.

\section{Acknowledgments}

This study was supported by the Basic Public Welfare Research Program of Zhejiang Province (no. LGF18H040001) 
and the Ningbo Social Development Major Project (no. 2017C510009).

\section{Supplementary Materials}

\author{
Supplementary 1. Figure S1. Study design.
}

Supplementary 2. Figure S2. The correlation between VCAN expression and the 4-miRNAs signature expression in the TCGA dataset.

Supplementary 3. Figure S3. The correlation between TGF $\beta$ expression and the 4-miRNAs signature expression in the TCGA dataset.

Supplementary 4. Table S1. Master regulated analysis results

Supplementary 5. Table S2. Patients risk stratification

\section{References}

[1] U. A. Matulonis, A. K. Sood, L. Fallowfield, B. E. Howitt, J. Sehouli, and B. Y. Karlan, "Ovarian cancer," Nature Reviews Disease Primers, vol. 2, article 16061, 2016.

[2] G. C. Jayson, E. C. Kohn, H. C. Kitchener, and J. A. Ledermann, "Ovarian cancer," The Lancet, vol. 384, no. 9951, pp. 1376-1388, 2014.

[3] A. N. Karnezis, K. R. Cho, C. B. Gilks, C. L. Pearce, and D. G. Huntsman, "The disparate origins of ovarian cancers: Pathogenesis and prevention strategies," Nature Reviews Cancer, vol. 17, no. 1, pp. 65-74, 2017.

[4] R. L. Siegel, K. D. Miller, and A. Jemal, "Cancer statistics, 2015," CA: A Cancer Journal for Clinicians, vol. 65, no. 1, pp. 5-29, 2015.

[5] J. Kang, A. D. D’Andrea, and D. Kozono, "A DNA repair pathway-focused score for prediction of outcomes in ovarian cancer treated with platinum-based chemotherapy," Journal of the National Cancer Institute, vol. 104, no. 9, pp. 670-681, 2012.

[6] T. Z. Tan, Q. H. Miow, R. Y.-J. Huang et al., "Functional genomics identifies five distinct molecular subtypes with clinical relevance and pathways for growth control in epithelial ovarian cancer," EMBO Molecular Medicine, vol. 5, no. 7, pp. 983-998, 2013.

[7] M. Riester, W. Wei, L. Waldron et al., "Risk prediction for latestage ovarian cancer by meta-analysis of 1525 patient samples," Journal of the National Cancer Institute, vol. 106, no. 5, Article ID dju048, 2014.

[8] R. W. Tothill, A. V. Tinker, J. George et al., "Novel molecular subtypes of serous and endometrioid ovarian cancer linked to clinical outcome," Clinical Cancer Research, vol. 14, no. 16, pp. 5198-5208, 2008.

[9] Cancer Genome Atlas Research Network, "Integrated genomic analyses of ovarian carcinoma," Nature, vol. 474, pp. 609-615, 2011.

[10] G. E. Konecny, C. Wang, H. Hamidi, B. Winterhoff, K. R. Kalli, J. Dering et al., "Prognostic and therapeutic relevance of molecular subtypes in high-grade serous ovarian cancer," JNCI: Journal of the National Cancer Institute, vol. 106, no. 10, 2014.

[11] R. Rupaimoole and F. J. Slack, "MicroRNA therapeutics: towards a new era for the management of cancer and other diseases," Nature Reviews Drug Discovery, vol. 16, no. 3, pp. 203221, 2017.

[12] A. H. F. Mirnezami, K. Pickard, L. Zhang, J. N. Primrose, and G. Packham, "MicroRNAs: key players in carcinogenesis and novel therapeutic targets," European Journal of Surgical Oncology, vol. 35, no. 4, pp. 339-347, 2009.

[13] M. V. Iorio, R. Visone, G. Di Leva et al., "MicroRNA signatures in human ovarian cancer," Cancer Research, vol. 67, no. 18, pp. 8699-8707, 2007.

[14] A. Vecchione, B. Belletti, F. Lovat et al., "A microRNA signature defines chemoresistance in ovarian cancer through modulation of angiogenesis," Proceedings of the National Acadamy of Sciences of the United States of America, vol. 110, no. 24, pp. 98459850, 2013.

[15] M. Bagnoli, S. Canevari, D. Califano et al., "Development and validation of a microRNA-based signature (MiROvaR) to predict early relapse or progression of epithelial ovarian cancer: a cohort study," The Lancet Oncology, vol. 17, no. 8, pp. 1137-1146, 2016.

[16] M. Bagnoli, L. De Cecco, A. Granata et al., "Identification of a chrXq27.3 microRNA cluster associated with early relapse in advanced stage ovarian cancer patients," Oncotarget, vol. 6, no. 12, article 9643, 2015.

[17] D. Sean and P. S. Meltzer, "GEOquery: a bridge between the Gene Expression Omnibus (GEO) and BioConductor," Bioinformatics, vol. 23, no. 14, pp. 1846-1847, 2007.

[18] A. Colaprico, T. C. Silva, C. Olsen et al., "TCGAbiolinks: an R/Bioconductor package for integrative analysis of TCGA data," Nucleic Acids Research, vol. 44, no. 8, p. e71, 2016.

[19] M. E. Ritchie, B. Phipson, D. Wu et al., "Limma powers differential expression analyses for RNA-sequencing and microarray studies," Nucleic Acids Research, vol. 43, article e47, 2015.

[20] M. N. Fletcher, M. A. Castro, X. Wang et al., "Master regulators of FGFR2 signalling and breast cancer risk," Nature Communications, vol. 4, article 2464, 2013.

[21] M. Zhao, L. Kong, Y. Liu, and H. Qu, "DbEMT: an epithelialmesenchymal transition associated gene resource," Scientific Reports, vol. 5, article 11459, 2015.

[22] E. Fessler, M. Jansen, F. De Sousa E Melo et al., "A multidimensional network approach reveals microRNAs as determinants of the mesenchymal colorectal cancer subtype," Oncogene, vol. 35, no. 46, pp. 6026-6037, 2016.

[23] S.-P. Chen, B.-X. Liu, J. Xu et al., "MiR-449a suppresses the epithelial-mesenchymal transition and metastasis of hepatocellular carcinoma by multiple targets," BMC Cancer, vol. 15, article 706, 2015.

[24] L. Zhao, W. Wang, L. Xu et al., "Integrative network biology analysis identifies miR-508-3p as the determinant for the mesenchymal identity and a strong prognostic biomarker of ovarian cancer," Oncogene, vol. 38, no. 13, pp. 2305-2319, 2019.

[25] S. Josson, M. Gururajan, P. Hu et al., "miR-409-3p/-5p promotes tumorigenesis, epithelial-to-mesenchymal transition, and bone metastasis of human prostate cancer," Clinical Cancer Research, vol. 20, no. 17, pp. 4636-4646, 2014.

[26] G. Liu, D. Yang, R. Rupaimoole et al., "Augmentation of response to chemotherapy by microRNA-506 through regulation of RAD51 in serous ovarian cancers," JNCI: Journal of the National Cancer Institute, vol. 107, no. 7, 2015.

[27] T. May, J. M. Stewart, M. Q. Bernardini et al., “The prognostic value of perioperative, pre-systemic therapy CA125 levels in patients with high-grade serous ovarian cancer," International Journal of Gynecology \& Obstetrics, vol. 140, no. 2, pp. 247-252, 2018.

[28] R. W. Tothill, A. V. Tinker, J. George et al., "Novel molecular subtypes of serous and endometrioid ovarian cancer linked to 
clinical outcome," Clinical Cancer Research, vol. 14, no. 16, pp. 5198-5208, 2008.

[29] C. Jin, Y. Xue, Y. Li et al., "A 2-protein signature predicting clinical outcome in high-grade serous ovarian cancer," International Journal of Gynecologic Cancer, vol. 28, no. 1, pp. 51-58, 2018.

[30] J. Zhang, L. Yang, X. Xiang, Z. Li, K. Qu, and K. Li, "A panel of three oxidative stress-related genes predicts overall survival in ovarian cancer patients received platinum-based chemotherapy," Aging, vol. 10, no. 6, pp. 1366-1379, 2018.

[31] D. Yang, Y. Sun, L. Hu et al., "Integrated analyses identify a master microRNA regulatory network for the mesenchymal subtype in serous ovarian cancer," Cancer Cell, vol. 23, no. 2, pp. 186-199, 2013.

[32] L. Ceppi, S. Marchini, and R. Fruscio, "miRNA based signature for predicting epithelial ovarian cancer relapse-progression: a step forward to prime time clinical adoption?" Translational Cancer Research, vol. 5, no. S4, pp. S664-S667, 2016.

[33] Y. Lu, J. Lu, X. Li et al., "MiR-200a inhibits epithelialmesenchymal transition of pancreatic cancer stem cell," $B M C$ Cancer, vol. 14, article 85, 2014. 


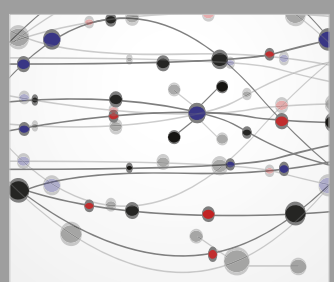

The Scientific World Journal
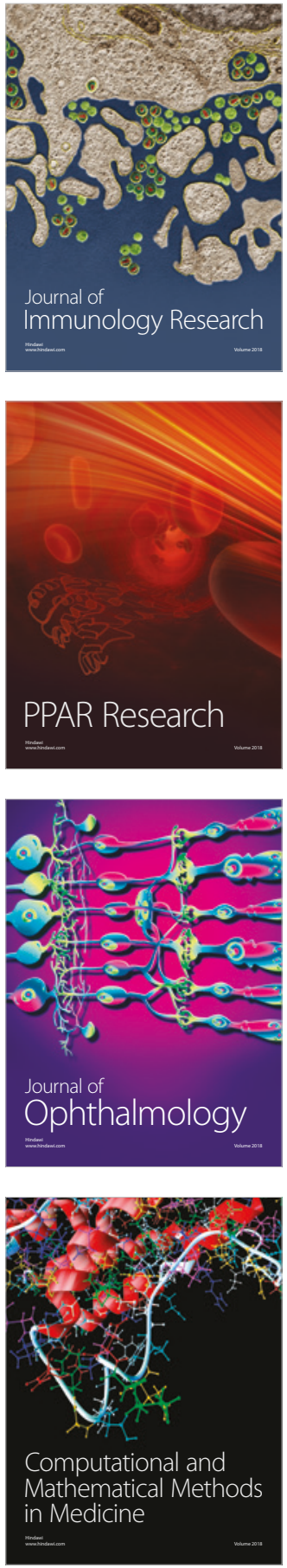

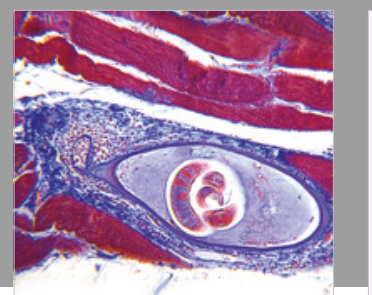

Gastroenterology Research and Practice

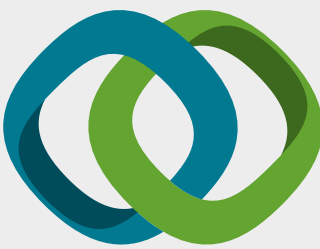

\section{Hindawi}

Submit your manuscripts at

www.hindawi.com
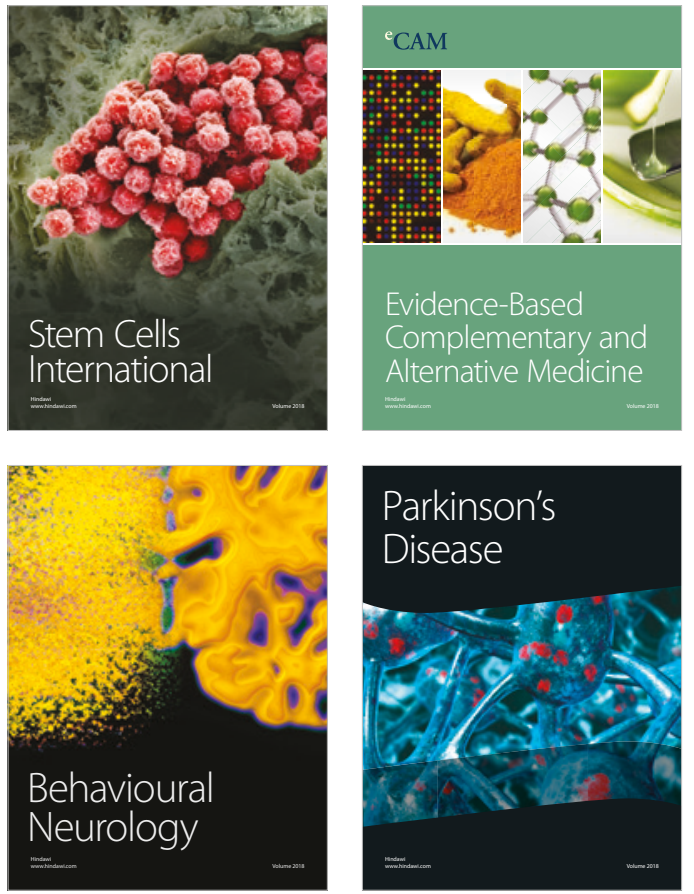

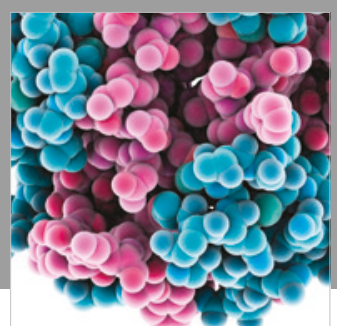

ournal of

Diabetes Research

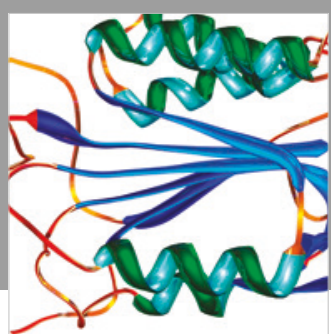

Disease Markers
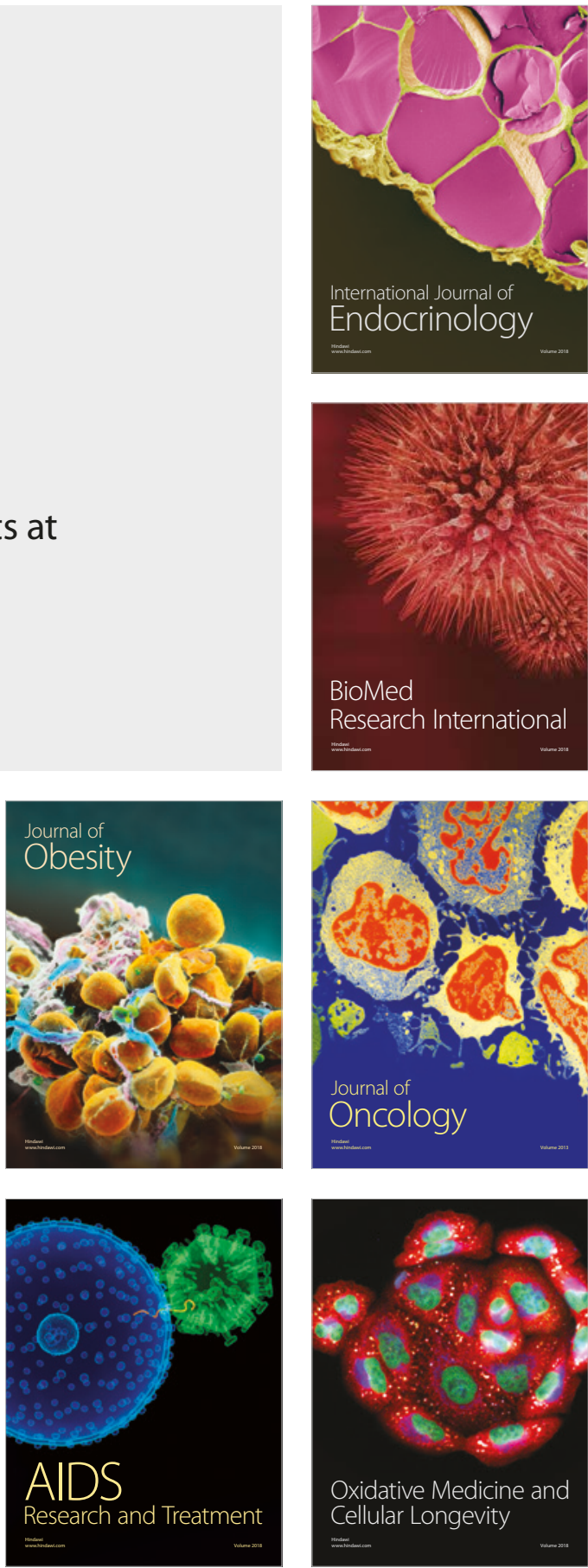